In the context class, we use "social group" (Q874405) from Wikidata. In the subject class, we use a new minted class "relative neocortex size" that is related to the class "size"(Q1152227) and "neocortex" (Q726562) from Wikidata. In the object class, we minted a new class "social group size" that is a subclass of "social group" (Q874405) from Wikidata.

\section{RDF code}

This is our formalization as a nanopublication in TriG format:

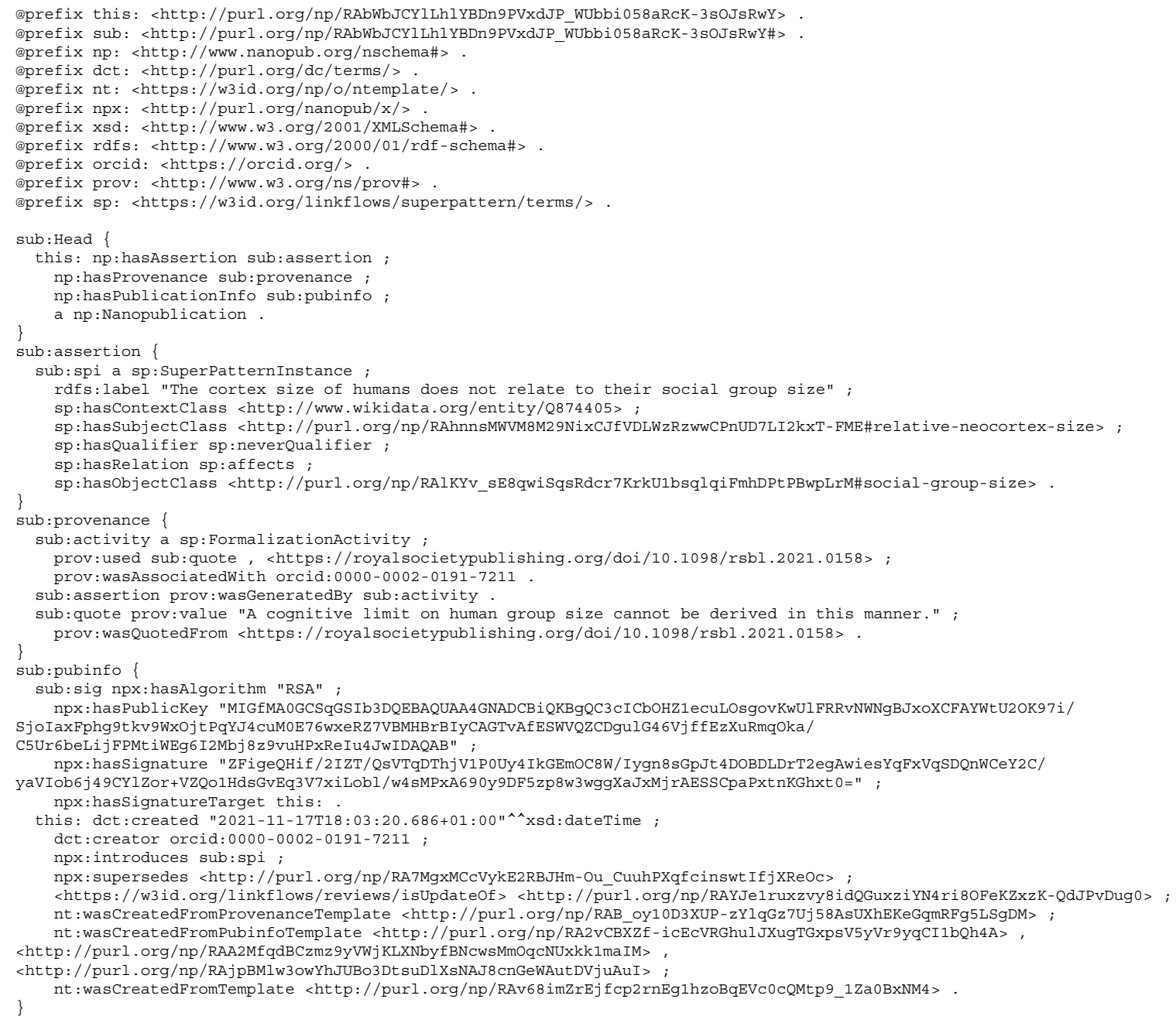

The following nanopublications introduce the newly minted classes in TriG format.

This is the class definition of "relative neocortex size":

@prefix this: <http://purl.org/np/RAhnnsMWVM8M2 9NixCJfVDLWzRzWWCPnUD7LI2kxT-FME> @prefix sub: <http://purl.org/np/RAhnnsMWVM8M2 9NixCJ fVDLWzRzWWCPnUD7LI2kxT-FME\#> @prefix np: <http://www. nanopub.org/nschema\#>

@prefix dct: <http://purl.org/dc/terms/>

@prefix nt: <https://w3id.org/np/o/ntemplate/>

@prefix npx: <http://purl.org/nanopub/x/> 


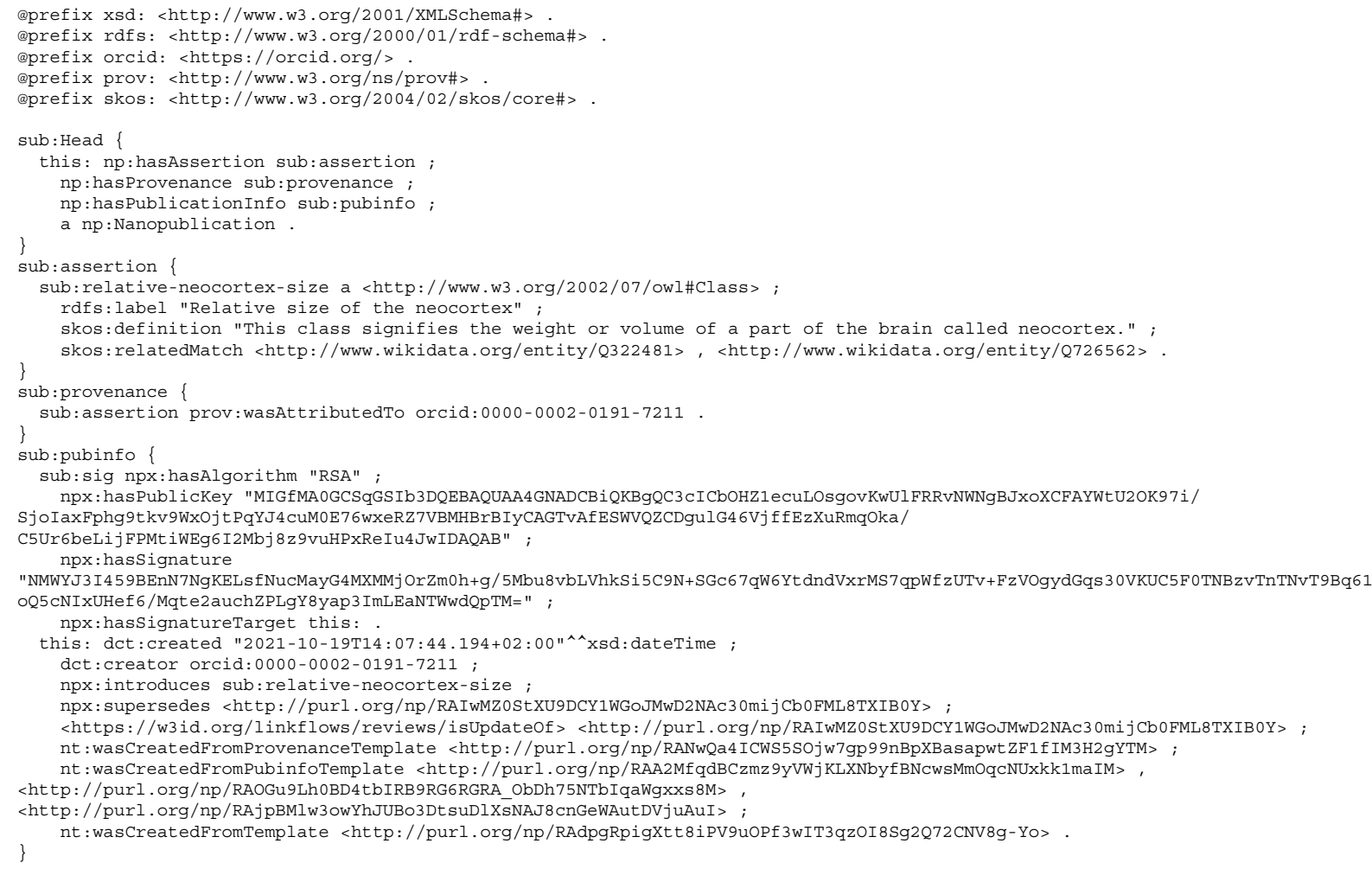

\section{This is the class definition of "social group size":}

@prefix this: <http://purl.org/np/RAlKYv_sE8qwiSqsRdcr7KrkU1bsqlqiFmhDPtPBwpLrM> @prefix sub: <http://purl.org/np/RAlKYv_sE8qwiSqsRdcr7KrkUlbsqlqiFmhDPtPBwpLrM\#> @prefix np: <http://www. nanopub.org/nschema\#>

@prefix dct: <http://purl.org/dc/terms/>

@prefix nt: <https://w3id.org/np/o/ntemplate/> .

@prefix npx: <http://purl.org/nanopub/x/>

@prefix xsd: <http://www.w3.org/2001/XMLSchema\#>

@prefix rdfs: <http://www.w3.org/2000/01/rdf-schema\#> .

@prefix orcid: <https://orcid.org/>

@prefix prov: <http://www.w3.org/ns/prov\#>

@prefix skos: <http://www.w3.org/2004/02/skos/core\#>

sub : Head \{

this: np:hasAssertion sub:assertion

$\mathrm{np}$ :hasProvenance sub:provenance ;

np:hasprovenance sub: provenance;

a np: Nanopublication

sub:assertion \{

sub: social-group-size a <http://www.w3.org/2002/07/owl\#Class>

rdfs:label "Social group size"

rdfs: subClassof <https://www.wikidata.org/wiki/Q874405>

skos:definition "Social group size is the size of a group of humans that a person socially interacts with (on a regular basis)." .

sub: provenance

sub:pubinfo \{

sub:sig npx:hasAlgorithm "RSA"

npx: hasPublicKey "MIGfMA0GCSqGSIb3DQEBAQUAA4 GNADCBiOKBgOC3 CICbOHZ1ecuLOsgovKwU1FRRvNWNgBJxoXCFAYWtU2OK97i/

SjoIaxFphg9tkv9WxOjtPqYJ4 cuM0E76 wxeRZ7VBMHBrBIYCAGTVAfESWVQZCDgulG46VjffEzXuRmqOka/

C5Ur6beLijFPMtiWEg6I2Mbj8z9vuHPxReIu4JWIDAQAB" ,

npx: hassignature

"WBZ10x+hSX0zXOXQAGBkM89SWDOnViMI 8NoLH8w1Gsj OWSj T/pasNugo6CEDZkWb5ZyHsXSSzG7JDCldnhVQkH64Nuok6C3QVVV9+EUVMZaDHBYMhObzJ3Pld8p

YNHWtW1 wNve4 Xkb91Mzq5zYfBw+Y4ufB5Kqi39zH1dr6XRZRo=" ;

npx: hassignatureTarget this:

this: dct:created "2021-06-22T09:49:42.152Z"^^xsd:dateTime ; 
dct: creator orcid:0000-0002-0191-7211

npx: introduces sub:social-group-size;

nt: wasCreatedFromProvenanceTemplate <http://purl.org/np/RANwQa4ICWS5SOjw7gp99nBpXBasapwtZF1fIM3H2gYTM> ; nt:wasCreatedFromPubinfoTemplate <http://purl.org/np/RAA2MfqdBCzmz9yVWj KLXNbyfBNCwSMmOqcNUxkk1maIM>

nt: wasCreatedFromTemplate <http://purl.org/np/RAdpgRpigXtt8iPV9uOPf3wIT3qzOI8Sg2Q72CNV8g-Yo> .

\section{References}

[1] C.I. Bucur, T. Kuhn, D. Ceolin and J. van Ossenbruggen, Expressing high-level scientific claims with formal semantics, in: Proceedings of the 11th Knowledge Capture Conference 2021, 2021. doi:10.1145/3460210.3493561.

[2] P. Lindenfors, A. Wartel and J. Lind, 'Dunbar's number' deconstructed, Biol. Lett. (2021). doi:10.1098/rsbl.2021.0158. 